\title{
Genome-Wide Association Studies Associated with Backfat Thickness in Landrace and Yorkshire Pigs
}

\author{
Young-Sup Lee, Donghyun Shin* \\ Department of Animal Biotechnology, Chonbuk National University, Jeonju 54896, Korea
}

\begin{abstract}
Although pork quality traits are important commercially, genome-wide association studies (GWASs) have not well considered Landrace and Yorkshire pigs worldwide. Landrace and Yorkshire pigs are important pork-providing breeds. Although quantitative trait loci of pigs are well-developed, significant genes in GWASs of pigs in Korea must be studied. Through a GWAS using the PLINK program, study of the significant genes in Korean pigs was performed. We conducted a GWAS and surveyed the gene ontology $(\mathrm{GO})$ terms associated with the backfat thickness (BF) trait of these pigs. We included the breed information (Yorkshire and Landrace pigs) as a covariate. The significant genes after false discovery rate $(<0.01)$ correction were AFG1L, SCAI, RIMS1, and SPDEF. The major GO terms for the top $5 \%$ of genes were related to neuronal genes, cell morphogenesis and actin cytoskeleton organization. The neuronal genes were previously reported as being associated with backfat thickness. However, the genes in our results were novel, and they included ZNF280D, BAIAP2, LRTM2, GABRA5, $P C D H 15, H E R C 1, D T N B P 1, S L I T 2, T R A P P C 9, N G F R$, APBB2, RBPJ, and ABL2. These novel genes might have roles in important cellular and physiological functions related to BF accumulation. The genes related to cell morphogenesis were NOX4, MKLN1, ZNF280D, BAIAP2, DNAAF1, LRTM2, PCDH15, NGFR, RBPJ, MYH9, APBB2, DTNBP1, TRIM62, and SLIT2. The genes that belonged to actin cytoskeleton organization were MKLN1, BAIAP2, PCDH15, BCAS3, MYH9, DTNBP1, ABL2, ADD2, and SLIT2.
\end{abstract}

Keywords: backfat thickness, genome-wide association studies, Landrace, neuronal gene, Yorkshire

\section{Introduction}

Landrace and Yorkshire pigs are commercial breeds used for pork production. The Landrace pig is a long, white pig with 16 or 17 ribs. Landrace pigs are utilized as Grandparents in the production of F1 parent stock females in a terminal crossbreeding (http://nationalswine.com/about/about_breeds/ landrace.php). They outperform other pigs in litter size, birth and weaning weight, rebreeding interval, longevity and durability. Yorkshire pigs are muscular with a high proportion of lean meat. Yorkshire pigs have been maintained with great diligence, with a focus on sow productivity, growth, and backfat formation.

In Korea, the Landrace and Yorkshire breeds in Korea are used for commercial pork production. Pork quality traits are polygenic and quantitative trait loci (QTL) are associated with pork quality traits. In this regard, we analyzed genome- wide association studies (GWASs) for backfat thickness (BF) for these breeds. BF is an important trait in pork production. Despite the importance of $\mathrm{BF}$, there have not been in-depth GWAS of the BF in the Landrace and Yorkshire breeds.

Rohrer et al. [1] performed a genome scan for loci affecting pork quality including BF in a Duroc-Landrace F2 population. Okumura et al. [2] studied genomic regions affecting BF by GWAS in a Duroc pig population. Likewise, we performed a GWAS and surveyed gene ontology (GO) terms. The top $5 \%$ genes were selected for GO analysis after the GWAS. The GO terms of the top $5 \%$ genes belonged to major GO terms of neuronal genes, cell morphogenesis and actin cytoskeleton organization [3-5]. Lee et al. [4] used a GWAS for fat thickness in humans and pigs and found that neuronal genes were associated with fat thickness. The results of our study were similar but the genes significantly associated with BF were novel. 


\section{Methods}

\section{Data preparation}

The number of Landrace individuals was 1,041 and the number of Yorkshire individuals was 836. Their BF were measured and the genomic DNA of each individual Landrace or Yorkshire pig was genotyped using an Illumina Porcine 60 K SNP Beadchip (Illumina, San Diego, CA, USA). Total number of single nucleotide polymorphisms (SNPs) was 62,551 and 62,551 in the Landrace and Yorkshire breeds, respectively. After quality control (minor allele frequency $<0.05$, Hardy-Weinberg equilibrium p $<0.0001$ and genotyping rate threshold $<0.05)$, the number of SNPs in the Landrace and Yorkshire breeds was 42,654 and 57,799, respectively. After merging the data of the two breeds, the number of SNPs was 38,002.

\section{GWAS of BF}

A GWAS using BF of Landrace and Yorkshire pigs was performed. The PLINK program was used for the GWAS [6]. The covariates were sex, parity and breed. The model used for the Landrace and Yorkshire pigs was the following:

$$
y_{i}=\mu+\text { sex }+ \text { parity }+ \text { breeds }+\beta^{*} g_{i}+e_{i},
$$

Table 1. Summary statistics of backfat thickness in the two pig breeds

\begin{tabular}{ccccc}
\hline Breed & Min & Max & Average & $\begin{array}{c}\text { Standard } \\
\text { deviation }\end{array}$ \\
\hline Landrace & 7.22 & 19.42 & 11.89 & 1.54 \\
Yorkshire & 8.44 & 18.04 & 12.70 & 1.49 \\
\hline
\end{tabular}

where $y_{i}$ is the backfat thickness of the $\mathrm{i}$-th individual, $\mu$ is the mean of backfat thickness in the population, sex, parity and breeds are the covariate, $\beta$ is the beta effect in GWAS, $g_{i}$ represents the SNP genotypes and $e_{i}$ is the error in the model.

\section{GO analysis}

We analyzed the GO of the top 5\% genes (p-value order) associated with BF in Landrace and Yorkshire pigs. Because the test result with a p-value $<0.05$ in our study was very genial and multiple testing such as the Bonferroni correction and the false discovery rate (FDR) of our study result was very restrictive, we performed the GO analysis with the top $5 \%$ genes. The gene catalogue was retrieved from Ensemble database (http://www.ensembl.org) and the GO analysis was performed with the DAVID (david.ncifcrf.gov; Database for Annotation, Visualization and Integrated Discovery v6.7). A list of gene identifiers was uploaded to summarize the functional annotations associated with groups or each individual genes and each biological process-related GO terms was based on the number of genes and the $p$-values $[7,8]$.

\section{Results \\ Phenotype description}

The BF statistics for Landrace pigs were 7.22 (minimum), 19.42 (maximum), 11.89 (average), and 1.54 (standard deviation). The BF statistics for Yorkshire pigs were 8.44 (minimum), 18.04 (maximum), 12.70 (average), and 1.49 (standard deviation). The average value of Yorkshire BF was greater than that of Landrace $\mathrm{BF}$, while the maximum value of Yorkshire BF was less than that of Landrace pig BF. Table 1

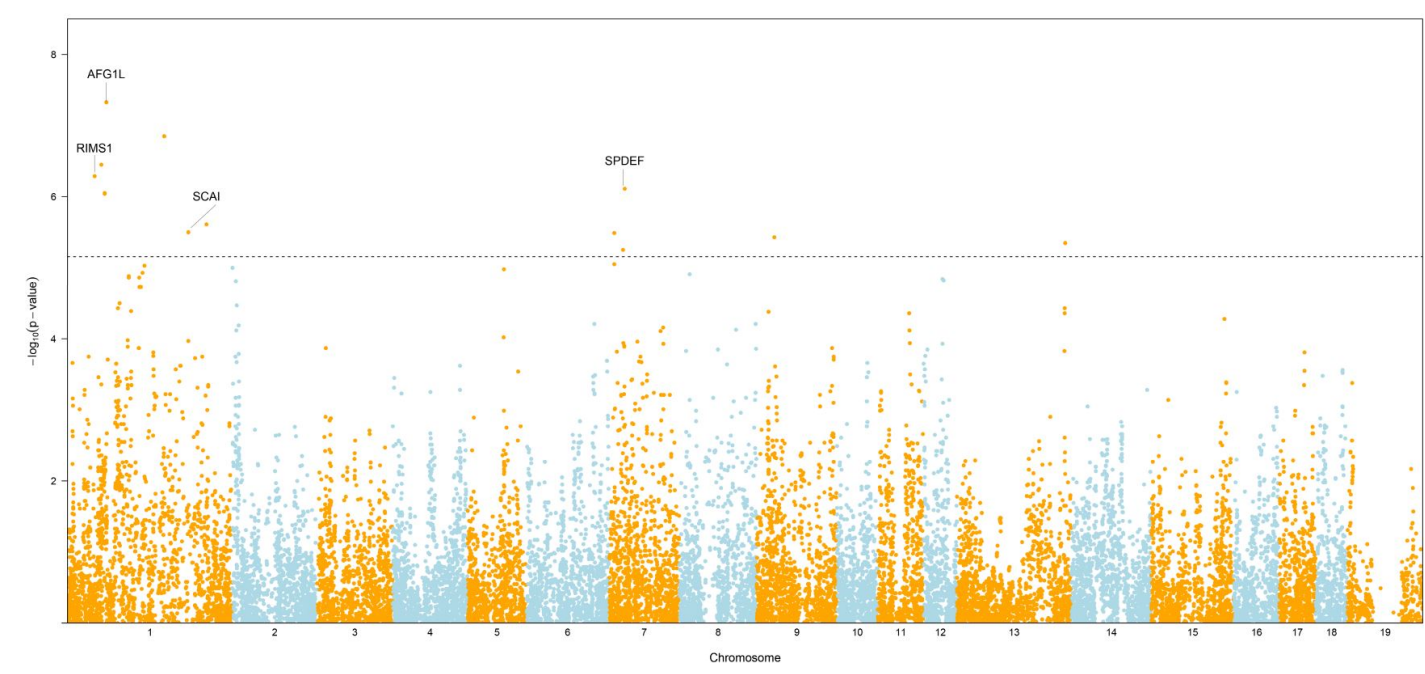

Fig. 1. Mahattan plot showing the $-\log _{10}($ p-value $)$ for backfat thickness across chromosomes in Landrace and Yorkshire pigs in Korea. The horizontal dotted line represents an false discovery rate of 0.01 . 
shows the BF summary statistics of these two breeds in Korea.

\section{GWAS in Landrace and Yorkshire pigs}

The GWAS was performed and the p-values were FDR-corrected. Fig. 1 shows the Manhattan plot across chromosomes. In our analysis, SNPs on chromosome 1 were frequent and the SCAI, AFG1L, and RIMS1 genes on chromosome 1, SPDEF on chromosome 7 were the most significant genes after FDR correction. The SCAI gene is a suppressor of cancer cell invasion. AFG1L is an ATPase family gene 1 homolog. RIMS1 is a member of the Ras superfamily of genes and a synaptic protein that regulates synaptic vesicle exocytosis. SPDEF encodes SAM pointed domain-containing ETS transcription factor. The boxplots of the sex and parity covariates in our model are shown (Figs. 2, $3)$. The figures show that females had greater BF than males. Table 2 shows the significant SNPs and those containing genes using an FDR cutoff p-value of 0.01 . Most of the significant SNPs had a positive beta effect. Fig. 4 represents the quantile-quantile plot (QQ-plot) of GWAS p-values and it shows the normality of our results. Fig. 5 illustrates the principal component analysis in Landrace and Yorkshire breeds to check the population stratification problem between Landrace and Yorkshire samples. It shows separation between Landrace and Yorkshire pigs in PC1 (Principal Component 1).

\section{GO analysis}

A GO analysis was performed in Landrace and Yorkshire pigs in Korea. Table 3 shows the GO terms with a p-value $<$ 0.05. The major GO terms were related to neuronal genes, cell morphogenesis and actin cytoskeleton organization and reorganization. Neuronal genes were reported to be related to subcutaneous fat thickness in human and pig [4]. However, the neuronal genes mentioned above did not match any genes in our neuron-related genes. The neuronal
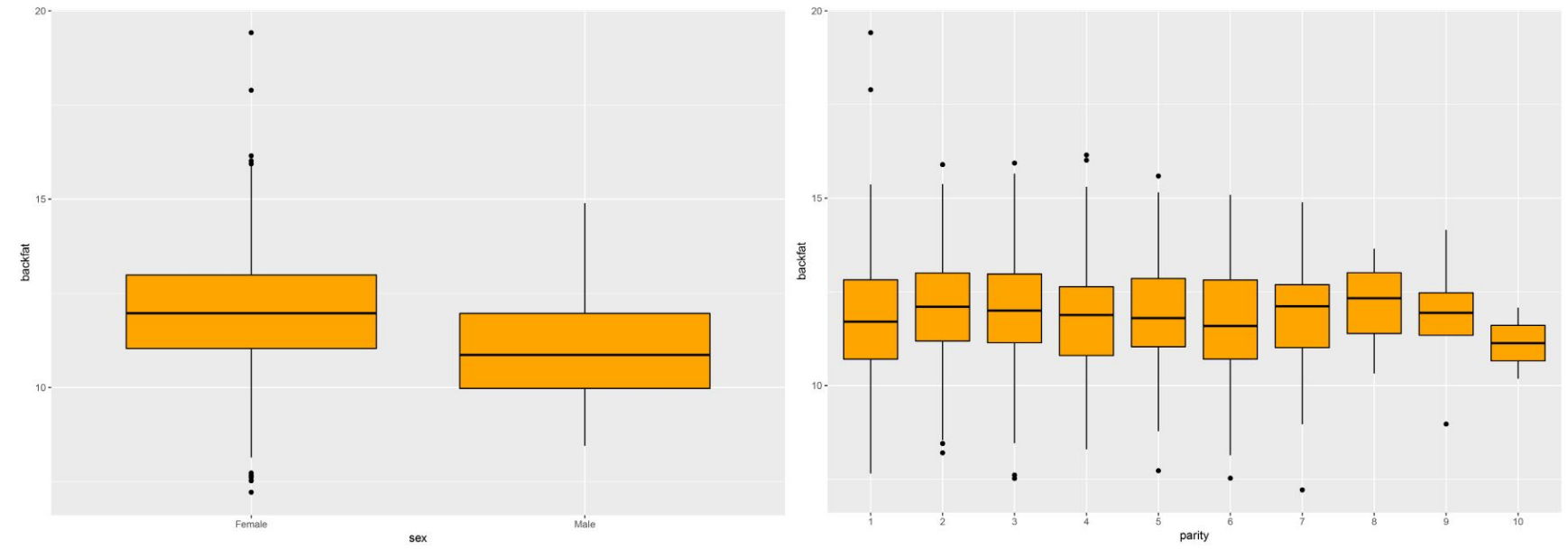

Fig. 2. Boxplots of sex and parity in Landrace pigs.
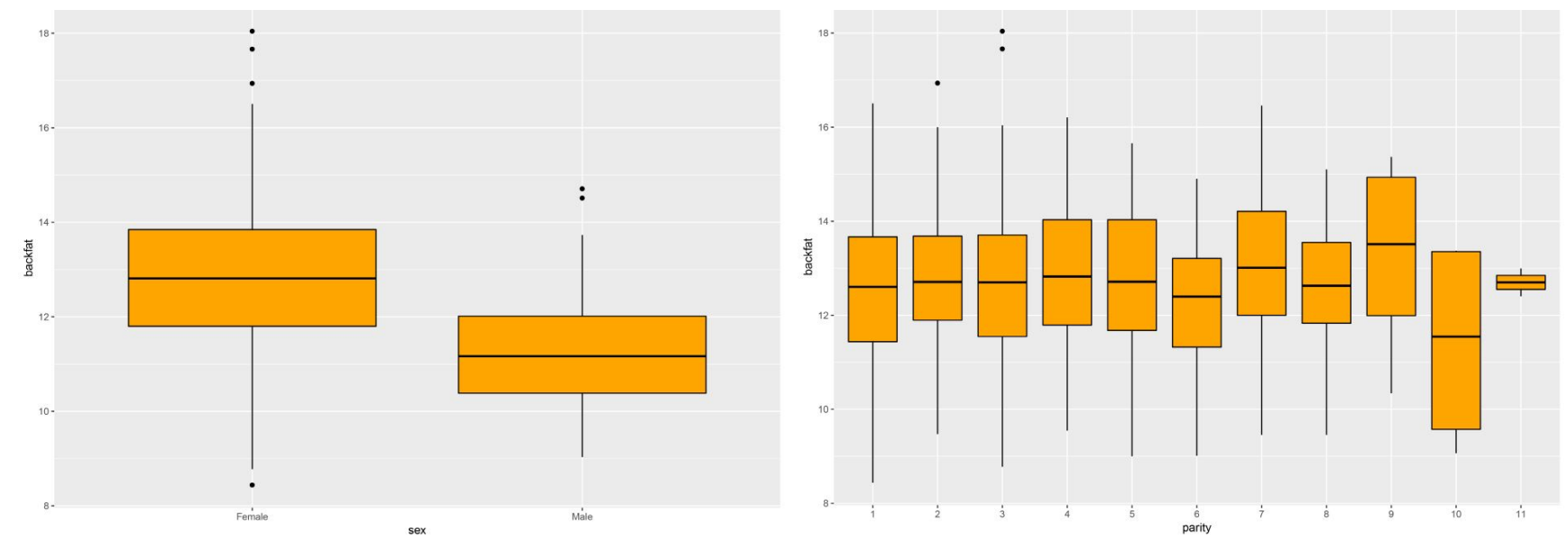

Fig. 3. Boxplots of sex and parity in Yorkshire pigs. 
YS Lee and D Shin. Genome-Wide Association Studies

Table 2. The significant SNPs and those-containing genes associated with BF after FDR correction

\begin{tabular}{|c|c|c|c|c|c|}
\hline Chr & SNP & Base pair & Beta & FDR & Gene \\
\hline 1 & ALGA0003992 & $74,391,668$ & 0.5628 & 0.0010 & AFG1L \\
\hline 1 & ALGA0006854 & $185,048,329$ & 0.5478 & 0.0016 & \\
\hline 1 & ALGA0003716 & $65,086,290$ & 0.4237 & 0.0027 & \\
\hline 1 & ALGA0003091 & $52,127,592$ & 0.7812 & 0.0029 & RIMS1 \\
\hline 7 & ASGA0032161 & $30,521,413$ & 0.6079 & 0.0029 & SPDEF \\
\hline 1 & CASI0007774 & $71,401,555$ & 0.4248 & 0.0029 & \\
\hline 1 & H3GA0001881 & $71,455,634$ & 0.4248 & 0.0029 & \\
\hline 1 & DRGA0002197 & $265,831,621$ & 0.3553 & 0.0069 & SCAI \\
\hline 1 & MARC0031246 & $231,303,178$ & 0.4662 & 0.0072 & \\
\hline 7 & ASGA0031143 & $10,707,229$ & -0.3348 & 0.0072 & \\
\hline 9 & ALGA0104955 & $33,658,036$ & -0.348 & 0.0076 & \\
\hline 13 & MARC0056987 & $206,756,876$ & 0.3341 & 0.0084 & \\
\hline 7 & ASGA0031992 & $27,352,011$ & 0.3849 & 0.0097 & \\
\hline
\end{tabular}

Most of the significant SNPs belonged to chromosome 1.

SNP, single nucleotide polymorphism; BF, backfat thickness; FDR, false discovery rate; AFG1L, AFG1 like ATPase; RIMS1, regulating synaptic membrane exocytosis 1; SPDEF, SAM pointed domain containing ETS transcription factor; SCAI, suppressor of cancer cell invasion.

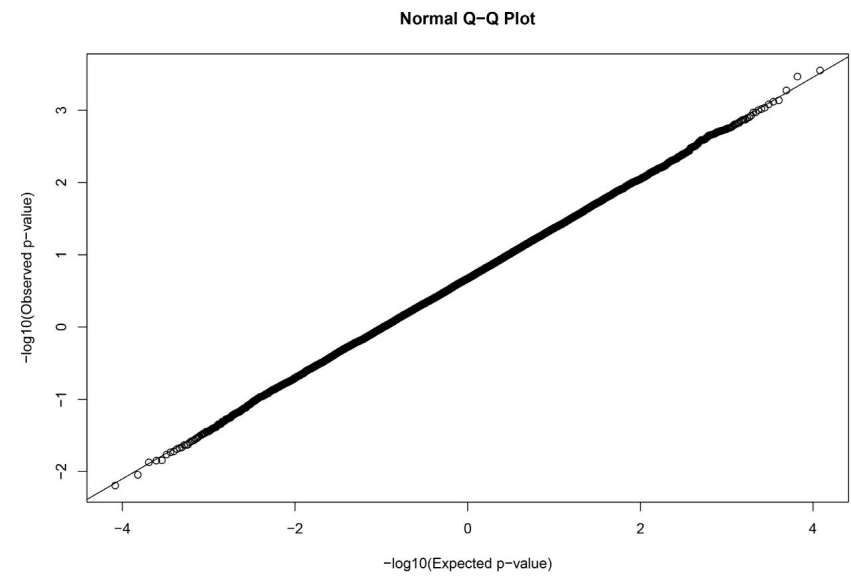

Fig. 4. The quantile-quantile plot (QQ-plot) of genome-wide association study $\mathrm{p}$-values. It represents the normality of our results.

genes were ZNF280D, BAIAP2, LRTM2, GABRA5, PCDH15, HERC1, DTNBP1, SLIT2, TRAPPC9, NGFR, APBB2, RBPJ, and $A B L 2$. We considered the association between fat accumulation and these neuronal genes to be novel. The cell morphogenesis genes were NOX4, MKLN1, ZNF280D, BAIAP2, DNAAF1, LRTM2, PCDH15, NGFR, RBPJ, MYH9, APBB2, DTNBP1, TRIM62, and SLIT2. In addition, the actin cytoskeleton organization and reorganization genes were MKLN1, BAIAP2, PCDH15, BCAS3, MYH9, DTNBP1, ABL2, ADD2, and SLIT2. Among these genes, PCDH15 is related to individual noise-induced hearing loss (NIHL) susceptibility. NIHL is a commonly recorded disorder, accounting for $7 \%$ to $21 \%$ of hearing loss [9]. NGFR is the nerve growth factor receptor [10] and dystrobrevin-binding protein 1 (DTNBP1) is an NMDA-receptor mediated signaling gene. NMDA is

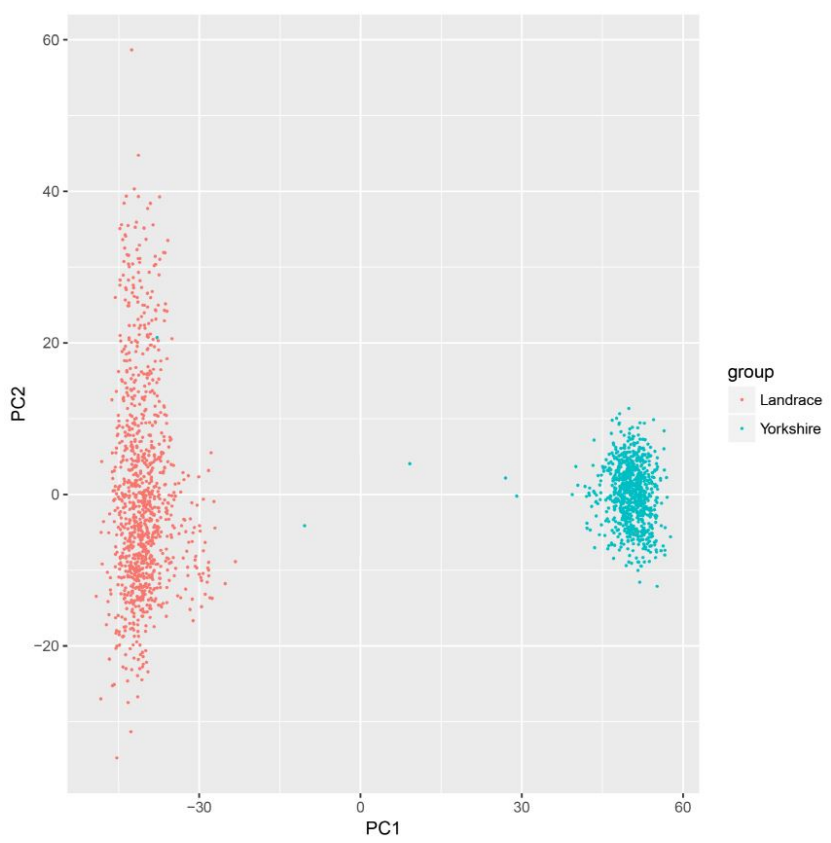

Fig. 5. Principal component analysis in Landrace and Yorkshire pigs to check the population stratification problem between Landrace and Yorkshire pigs.

N-methyl-D-aspartate and DTNBP1 has the ability to modulate synaptic plasticity and glutamatergic transmission through NMDA receptors [11]. BAIAP2 is the brain-specific angiogenesis inhibitor-1 associated protein gene [12] 
Table 3. GO terms of the top $5 \%$ genes associated with backfat thickness

\begin{tabular}{|c|c|c|c|c|}
\hline Term & Count & p-value & Gene & Fold enrichment \\
\hline $\begin{array}{l}\text { GO:0031532 actin cytoskeleton } \\
\text { reorganization }\end{array}$ & 5 & $8.26 \mathrm{E}-04$ & MKLN1, BAIAP2, BCAS3, MYH9, DTNBP1 & 11.67 \\
\hline GO:0040011 locomotion & 15 & 0.01 & $\begin{array}{l}\text { ZNF280D, ITGA11, SCAI, DOCK8, CD151, } \\
\text { MYH9, RUFY3, SLIT2, PTK2, NGFR, BCAS3, } \\
\text { APBB2, ABL2, CLN6, CYP19A1 }\end{array}$ & 2.03 \\
\hline $\begin{array}{l}\text { GO:0030036 actin cytoskeleton } \\
\text { organization }\end{array}$ & 9 & 0.01 & $\begin{array}{l}\text { MKLN1, BAIAP2, PCDH15, BCAS3, MYH9, } \\
\text { DTNBP1, ABL2, ADD2, SLIT2 }\end{array}$ & 2.90 \\
\hline GO:0000902 cell morphogenesis & 14 & 0.01 & $\begin{array}{l}\text { NOX4, MKLN1, ZNF280D, BAIAP2, DNAAF1, } \\
\text { LRTM2, PCDH15, NGFR, RBPI, MYH9, } \\
\text { APBB2, DTNBP1, TRIM62, SLIT2 }\end{array}$ & 2.11 \\
\hline $\begin{array}{l}\text { GO:0000904 cell morphogenesis } \\
\text { involved in differentiation }\end{array}$ & 10 & 0.02 & $\begin{array}{l}\text { ZNF280D, LRTM2, PCDH15, NGFR, RBPJ, } \\
\text { MYH9, APBB2, DTNBP1, TRIM62, SLIT2 }\end{array}$ & 2.55 \\
\hline $\begin{array}{l}\text { GO: } 1901187 \sim \text { regulation of ephrin } \\
\text { receptor signaling pathway }\end{array}$ & 2 & 0.02 & ANKS1A, RBPJ & 107.38 \\
\hline GO:0030182 neuron differentiation & 13 & 0.02 & $\begin{array}{l}\text { ZNF280D, BAIAP2, LRTM2, GABRA5, } \\
\text { PCDH15, HERC1, DTNBP1, SLIT2, TRAPPC9, } \\
\text { NGFR, APBB2, RBPJ, ABL2 }\end{array}$ & 2.11 \\
\hline GO:0048666 neuron development & 11 & 0.02 & $\begin{array}{l}\text { ZNF280D, BAIAP2, GABRA5, LRTM2, } \\
\text { PCDH15, NGFR, HERC1, APBB2, DTNBP1, } \\
\text { ABL2, SLIT2 }\end{array}$ & 2.29 \\
\hline $\begin{array}{l}\text { GO:0032989 cellular component } \\
\text { morphogenesis }\end{array}$ & 14 & 0.02 & $\begin{array}{l}\text { NOX4, MKLN1, ZNF280D, BAIAP2, DNAAF1, } \\
\text { LRTM2, PCDH15, NGFR, RBPJ, MYH9, } \\
\text { APBB2, DTNBP1, TRIM62, SLIT2 }\end{array}$ & 1.99 \\
\hline $\begin{array}{l}\text { GO:0050804 modulation of synaptic } \\
\text { transmission }\end{array}$ & 5 & 0.02 & GRM4, BAIAP2, RIN1, DTNBP1, RIMS4 & 4.67 \\
\hline $\begin{array}{l}\text { GO:0030030 cell projection } \\
\text { organization }\end{array}$ & 13 & 0.02 & $\begin{array}{l}\text { ZNF280D, BAIAP2, DNAAF1, LRTM2, } \\
\text { PCDH15, NGFR, BCAS3, MYH9, HERC1, } \\
\text { APBB2, DTNBP1, ABL2, SLIT2 }\end{array}$ & 2.03 \\
\hline $\begin{array}{l}\text { GO:0030029 actin filament-based } \\
\text { process }\end{array}$ & 9 & 0.03 & $\begin{array}{l}\text { MKLN1, BAIAP2, PCDH15, BCAS3, MYH9, } \\
\text { DTNBP1, ABL2, ADD2, SLIT2 }\end{array}$ & 2.51 \\
\hline $\begin{array}{l}\text { GO:0010455 positive regulation of } \\
\text { cell fate commitment }\end{array}$ & 2 & 0.03 & $S P D E F, R B P J$ & 71.59 \\
\hline GO:0048699 generation of neurons & 13 & 0.04 & $\begin{array}{l}\text { ZNF280D, BAIAP2, LRTM2, GABRA5, } \\
\text { PCDH15, HERC1, DTNBP1, SLIT2, TRAPPC9, } \\
\text { NGFR, APBB2, RBPJ, ABL2 }\end{array}$ & 1.90 \\
\hline $\begin{array}{l}\text { GO:0048667 cell morphogenesis } \\
\text { involved in neuron differentiation }\end{array}$ & 7 & 0.04 & $\begin{array}{l}\text { ZNF280D, LRTM2, PCDH15, NGFR, APBB2, } \\
\text { DTNBP1, SLIT2 }\end{array}$ & 2.77 \\
\hline $\begin{array}{l}\text { GO:1903047 mitotic cell cycle } \\
\text { process }\end{array}$ & 8 & 0.04 & $\begin{array}{l}\text { ANKRD17, SIN3A, TUBGCP5, USP8, RAD21, } \\
\text { CCNY, CAMK2D, CENPE }\end{array}$ & 2.48 \\
\hline $\begin{array}{l}\text { GO:0031175 neuron projection } \\
\text { development }\end{array}$ & 9 & 0.04 & $\begin{array}{l}\text { ZNF280D, BAIAP2, LRTM2, NGFR, HERC1, } \\
\text { APBB2, DTNBP1, ABL2, SLIT2 }\end{array}$ & 2.26 \\
\hline
\end{tabular}

The major gene ontology (GO) terms were neuron-related, cell morphogenesis, and actin cytoskeleton organization, and reorganization.

\section{Discussion}

\section{Genome-wide association studies}

The commercial pig breeds in Korea are mainly the Landrace, Yorkshire, Duroc and Berkshire breeds. Among these breeds, we analyzed GWASs of backfat thickness using the Landrace and Yorkshire breeds. Although there is a pig QTL database (QTL DB, https://www.animalgenome.org) associated with BF, we aimed to determine the significant variants and those containing genes by using the Korean pig population. While the QTL DB provides much information about QTL and significant genes, detailed information about the significant genes is not available. Thus, a GWAS should be performed with the QTL information.

\section{Neuron-related terms in the GO analysis}

It was reported that neuronal genes are closely related to fat accumulation [4]. Lee et al. [4] used local genomic sequencing and SNP association to identify genes for subcutaneous fat thickness. They reported that NEGR1, SLC44A5, PDE4B, LPHN2, ELTD1, ST6GALNAC5, and TTLL7 are fat-associated neuronal genes. However, in our analysis, 
these genes did not appear. This may be because the aforementioned study used Korean native pig. We examined $\mathrm{BF}$ in Korean Landrace pigs. Despite this difference, neuron-related terms were also major ones in our analysis. In Yorkshire pigs, neuronal genes were also found. The neuronal genes ZNF280D, BAIAP2, LRTM2, GABRA5, PCDH15, HERC1, DTNBP1, SLIT2, TRAPPC9, NGFR, APBB2, $R B P J$, and $A B L 2$ were not yet reported. In our study, these genes reported in the GWAS of pigs but they have been reported in GWASs of cattle, chickens, and sheep.

\section{Actin cytoskeleton organization and cell morphogenesis genes}

Among the actin cytoskeleton organization genes and cell morphogenesis genes, PCDH15, NGFR, DTNBP1, and BAIAP2 are also neuronal genes and thus can be classified into the $\mathrm{GO}$ terms of neuronal genes. These genes obviously play a role as neuronal genes, actin cytoskeleton organization genes (BAIAP2 gene) and cell morphogenesis genes (PCDH15, $N G F R$, and DTNBP1 genes). These genes not only play a role as neuronal genes but also act as actin cytoskeleton organization and cell morphogenesis genes. Interestingly, these neuronal genes are associated with brain function, nerves and diseases. Thus, some of the backfat-associated genes play a neuronal role. We expect that how backfat and neuronal genes or neuropathic genes are related to each other will be revealed.

ORCID: Young-Sup Lee: http://orcid.org/0000-0001-9267-0 850; Donghyun Shin: http://orcid.org/0000-0002-0819-0553

\section{Authors' contribution}

\author{
Conceptualization: YSL, DS \\ Data curation: DS \\ Formal analysis: YSL \\ Methodology: YSL, DS \\ Writing - original draft: YSL \\ Writing - review \& editing: YSL, DS
}

\section{Acknowledgments}

This research was supported by the Basic Science Research Program through the National Research Foundation of Korea (NRF) funded by the Ministry of Education (No. NRF-2017R1A6A3A11033784 \& NRF-2017R1C1B3007144).

\section{References}

1. Rohrer GA, Thallman RM, Shackelford S, Wheeler T, Koohmaraie M. A genome scan for loci affecting pork quality in a Duroc-Landrace F population. Anim Genet 2006;37:17-27.

2. Okumura N, Matsumoto T, Hayashi T, Hirose K, Fukawa K, Itou $\mathrm{T}$, et al. Genomic regions affecting backfat thickness and cannon bone circumference identified by genome-wide association study in a Duroc pig population. Anim Genet 2013;44: 454-457.

3. Muslin AJ. MAPK signalling in cardiovascular health and disease: molecular mechanisms and therapeutic targets. Clin Sci (Lond) 2008;115:203-218.

4. Lee KT, Byun MJ, Kang KS, Park EW, Lee SH, Cho S, et al. Neuronal genes for subcutaneous fat thickness in human and pig are identified by local genomic sequencing and combined SNP association study. PLoS One 2011;6:e16356.

5. Subramaniam S, Ozdener MH, Abdoul-Azize S, Saito K, Malik B, Maquart G, et al. ERK1/2 activation in human taste bud cells regulates fatty acid signaling and gustatory perception of fat in mice and humans. FASEB J 2016;30:3489-3500.

6. Purcell S, Neale B, Todd-Brown K, Thomas L, Ferreira MA, Bender D, et al. PLINK: a tool set for whole-genome association and population-based linkage analyses. Am J Hum Genet 2007;81:559-575.

7. Huang DW, Sherman BT, Tan Q, Kir J, Liu D, Bryant D, et al. DAVID Bioinformatics Resources: expanded annotation database and novel algorithms to better extract biology from large gene lists. Nucleic Acids Res 2007;35:W169-W175.

8. Moisa SJ, Shike DW, Shoup L, Loor JJ. Maternal plane of nutrition during late-gestation and weaning age alter steer calf longissimus muscle adipogenic microRNA and target gene expression. Lipids 2016;51:123-138.

9. Zong S, Zeng X, Liu T, Wan F, Luo P, Xiao H. Association of polymorphisms in heat shock protein 70 genes with the susceptibility to noise-induced hearing loss: a meta-analysis. PLoS One 2017;12:e188195.

10. Jayne T, Newman M, Verdile G, Sutherland G, Munch G, Musgrave I, et al. Evidence for and against a pathogenic role of reduced gamma-secretase activity in familial Alzheimer's disease. J Alzheimers Dis 2016;52:781-799.

11. Sacchetti E, Scassellati C, Minelli A, Valsecchi P, Bonvicini C, Pasqualetti P, et al. Schizophrenia susceptibility and NMDAreceptor mediated signalling: an association study involving 32 tagSNPs of DAO, DAOA, PPP3CC, and DTNBP1 genes. BMC Med Genet 2013;14:33.

12. Mori K, Kanemura Y, Fujikawa H, Nakano A, Ikemoto H, Ozaki I, et al. Brain-specific angiogenesis inhibitor 1 (BAI1) is expressed in human cerebral neuronal cells. Neurosci Res 2002;43:69-74. 